\title{
Study on Student's Decision-making Behavior Mode with Business Operations Simulation System through Protocol Analysis
}

\author{
Yu-Ling Lin, Hong-Wen Lin*, and Hui-Jun Chen
}

\begin{abstract}
This research is developed to study the information and considerations adopted by college and masterdegree students when operating the Business Operations Simulation System under various kinds of operation scenarios and the approaches used by them for integrating the variations of different business management knowledge. In this research, the protocol analysis is conducted to acquire the historical information of their mental activities, supported with experimental design to create the information recognition based comparative experiment to know how the decisions are made by students. Finally, the analysis and verification results are compared to obtain the behavioral features of students in problem solving and the process of information processing that can be physically described. According to the research, the results indicated that college students require more complicated thinking steps when solving the problem but their thinking elements required for decision-making are not as inclusive as master-degree students. Being less familiar with the issue of decision-making, the college students are normally unable to make correct decisions and work out the solutions; instead, they tend to proceed with thinking and judgment by intuitive method. Being more comprehensive in learning experiences and knowledge accumulation, the master-degree students are able to comprehend he provided information more quickly and they also develop specific kinds of solutions. Therefore, they are able to execute the decision and set up decision items in a more direct way.
\end{abstract}

Index Terms-Decision-making behavior, Protocol analysis, Problem solving, Simulation System.

\section{INTRODUCTION}

The purpose of education in modern days is to encourage the student to think independently and to develop innovative thinking so as to fortify their ability in decision-making and judgment in which, the teaching method focusing on competition is one of the main streams. Currently in Taiwan, many colleges are using the Business Operations Simulation System (BOSS) in their teaching and they also sponsored relevant contest activities to stimulate the knowledge exchange among students. In nature, the BOSS is a kind of comprehensive business management knowledge, which is

Published on October 5, 2019.

Yu-Ling Lin: Professor, Department of Business Administration, National Chin-Yi University of Technology, No.57, Sec. 2, Zhongshan Rd., Taiping Dist., Taichung 41170, Taiwan (R.O.C.)

Hong-Wen Lin (Corresponding Author): Assistant Professor, Department of International Trade, Chinese Culture University, No.55, Hwa-Kang Rd., Yang-Ming-Shan, Taipei 11114, Taiwan (R.O.C.) E-Mail: woodylin34@hotmail.com

Hui-Jun Chen: Undergraduate, Department of Business Administration, National Chin-Yi University of Technology, No.57, Sec. 2, Zhongshan Rd., Taiping Dist., Taichung 41170, Taiwan (R.O.C.) an educational game that allows students to increase the market share, net profit, product segmentation and bring down costs. In this regard, the BOSS has simulated the economic environment restrictions, the impact brought by the decision of competitors and all sorts of uncertainties that will be encountered during business operations. Based on the business operations-related information provided by the system, students will be able to make the respective decision in each round of game with the knowledge learned. For example, setting up production and materials quantity for production management; developing product price and activity strategies for marketing management; distribute the duty appointment when conducting the activities for human resource management; allocate $\mathrm{R} \& \mathrm{D}$ budget and maintenance expense budget for research and development management; and analyze the business financial statement for financial management in order to know the market conditions.

Decision-making refers to a process required to assess and select solutions according to the problem encountered and the anticipated result, and it represents a series of complicated thinking processes. However, the thinking process generated by the decision is easily ignored that it would be very difficult to express the decision thinking process clearly and completely. The ingredient of decision process is not a set of randomly stacked mechanical steps, and it requires a special method in order to understand the non-explicit knowledge. This research uses the protocol analysis as the foundation supported with experimental design in order to understand the ability and relevant knowledge that student are lacking during the decisionmaking process. In the meantime, it also provides educational guidance to teachers engaging in management education so that student may enjoy a more sophisticated training. In this research, the BOSS is based to study the difference between college and master-degree students in decision-making thinking process and problem solving.

\section{THEORETICAL BACKGROUND}

Until now, a lot of research programs have been developed to study the problem solving related behaviors, and the researches in previous also provide clear interpretation in defining the problem. In this regard, problem refers to a kind of scenario that should be achieved during which, it requires effective method and physical operations (Chi \& Glaser, 1985). According to Anderson (1990), Chi and Glaser (1985), they pointed out that the problem would usually comprise the following three 
common pre-conditions: Initial state: The professional knowledge or common knowledge initially owned by the individual together with the attitude and solution skills demonstrated when encountering the problem. Goal: The final goal or status to be achieved by the individual, which is also the final purpose of each individual problem. To successfully achieve the goal or simplify the entire process, the goal will be divided into several sub-goals in order to solve the problem smoothly. Operations: The activities used to change the original status and the goal, and it is usually defined between the above two natures. Further, Simon (1997) also pointed out that the state of problem presented inside if not provided by the problem itself; instead, it is structured by the problem solver in that the knowledge and the experience possessed by the problem solver will affect their comprehension to the problem. Even facing the same problem, the information required for each individual will not be the same. The information demand does not remain unchanged because it will be affected by all sorts of interfering factors (Leckie, Pettigrew, \& Sylvai, 1996). The factors causing the difference in information demand are job type, scholastic background or training, and information availability (Lin \& Garvey, 1972).

Problem solving is a process of efforts taken by the individual in order to achieve a goal without a fixed kind of solutions (Chi \& Glaser, 1985). Mayer (1992) explained that the problem solving represents a mental operating process required for the difficulties produced and problems encountered under a given state and target state. Regarding this, Vessey (1991) proposed that the cognitive fit phenomenon exists in the problem solving behavior exhibited by humans. In other words, when the problem solving auxiliary tools are compatible with the strategies required for supporting such mission, the complexity of the mission environment can be reduced, i.e. it would be much easier to solve the problem under cognitive fit conditions. The failure in problem solving is due to the mistake in problem solving direction and diagnostic approaches (MacDuffie, 1997).

Deek and McHugh (2000) considered that the problem solving process can be studied from subjective and objective points of views. As far as the subjective point of view is concerned, the individual needs to go through a series of subjective evolution in mental growth where the individual is always seeking the solutions starting from discovering the problem to working out the method. In an objective point of view, it means that the individual needs to understand the problem forming process from the original state to the target solution state when learning the solution. In problem solving, learning is not only an important process but is the comprehensive result through the cross-using of strategies and knowledge (Gagne, 1985). The knowledge will become useful by exactly applying the individual's professional skills or knowledge in problem solving process and it would be a meaningful learning (Mayer, 1992).

\section{Methodology}

\section{A. Research Steps}

To study the difference between college and masterdegree students in decision-making thinking process and problem solving, this research adopted the protocol analysis as the design method in order to proceed with the analysis of decision-making process later on.

The protocol analysis is a set of analysis methods applied during the message processing. Such analysis method is used to understand the thinking behavior process of human kinds in solving the problem, and it is mutually connected with cognitive psychology. To analyze the design thinking related issues more effectively and accurately, Eckersley (1988) studied the methodology for protocol analysis and proposed "verbal protocol" and "verbal encoding" should be the main analysis subjects. The data collection method, it is divided into "concurrent and retrospective" aspects. First, "concurrent" means that the respondents should use the "thinking aloud" method during the experiment process where they are asked to continuously express their thinking, solution process and decision-making behavior verbally in mental aspect. With the verbal data or other recordable information generated during the experiment process, such as graphical representation in sketches, the researcher analyzed the thinking process of the respondents in message treatment. The concurrent protocol analysis uses the message treatment theory as the basis by taking "design process" as the focus (Simon, 1992), therefore the "concurrent protocol analysis" is considered as more suitable for the "bottom-up" strategy when developing the design mode. Next, the "retrospective" is the status in which, the researcher inquire about the message accepting and expressing from the respondents according to the original record. As the "retrospective protocol analysis" puts focus on "the content of cognition," it is suitable for the "top-bottom" strategy.

When analyzing the decision-making process, the number is coded for the verbal information expressed by the respondents. As such, the thinking process of respondent's "queuing sequence of decision items" is divided into comprehension problems, plan, select and execution. In this way, the problem solving process is established according to the thinking process creating a sequence in order to understand the relationship between each sequence item.

\section{B. Experimental Design}

In the scenario of this research, the respondents are assumed as the general manager of a company competing in the market. By sequential order from $1 \sim 8$, the respondents are instructed to indicate the importance of these 8 business decision items (product pricing, planned production quantity, marketing budget, equipment investment budget, R\&D budget, quantity of material purchased, maintenance expense budget, stock dividend) in a BOSS according to their own experience or intuition. Later on, they are asked to explain the information that should be referenced in the following 6 financial statements (i.e. industrial background, market climate information, business situation statement, statement of cash flows, balance sheet, income statement) for the top-three business decision items, which are regarded as the most important by them. After the experiment, the respondents are also divided into two groups, i.e. college students and master-degree students, for studying the difference of these two groups in decision-making and referential information seeking. 
Regarding the experiment process, we employed the sound and video recording method in the research for recording the respondent's decision-making method during the entire process. First, the respondents are asked to fill out basic information. Next, we recorded the time required by the respondents in determining the sequence of these 8 decision-making items for use as the basis of calculating the thinking time of the respondents in order to understand the difference of the thinking speed between college students and master-degree students. In the meantime, we also recorded key information that should be referenced in these 6 financial statements for the top-three business decision items, which are regarded as the most important by them. In this way, we may understand that if they are able to use statements properly and use such duration as the basis for calculating their information reference time.

\section{Sampling and Participants}

In the sample collection aspect, the protocol analysis is focusing on the quality of the research information and not on massive amount of samples. By open recruiting method, we recruited the students who are majoring in business management department at the universities in Taiwan and they are composed of 15 college students and 12 masterdegree students. To avoid interference between students, only one respondent will be appointed to conduct the experiment each time. By using business functions as the specialty for distinguishing the realm of major, 17 are from marketing management, representing about $63 \% ; 1$ is from development management, representing about 3.7\%; 2 are from research \& development management, representing about $7.4 \%$; 4 are from financial management, representing about $14.8 \%$; and 2 are from others, representing about $11.1 \%$ (as shown in Table I).

\section{EXPERIMENTAL RESULTS}

\section{A. Analysis of Business Decision-making Process}

During the research, the sequential analysis is conducted for these 8 decision-making items recognized by 27 students (as shown in Table II). The results indicated that among the 8 business decision-making items, the R\&D budget is set by 9 students at the first priority because they considered that the R\&D budget would be crucial to the product quality where a solid base should be set for product quality in order to fortify the competitiveness; and the marketing budget is set by 11 students at the 2 nd position. The research result indicated that the marketing management is set by students as the most specialized expertise area and specialty background, but they did not set the marketing budget as the top priority decision-making item. Instead, they uphold that appropriate amount of marketing budget should be invested after launching the product or setting the product price in order to elevate the product awareness. Among them, 9 students set the quantity of material purchased at the 5th position and 7 of them set the planned production quantity at the 6th position. We also discovered that the thinking process of students is also arranged according to such sequential order where they concluded that the planned production quantity should be set after deciding the quantity of material purchased. In business decision-making sequence, we discovered that these students are sharing the same thinking elements in setting the product price that they set the product pricing at the 7 th position. It is because that they tend to set up the required budget beforehand and then evaluate the level of product pricing by which, the income and expenses can be determined. Among these 27 students, 23 of them set the stock dividend at the 8 th position. It is mainly that the dividend should be distributed when profit is earned by the company and that these students are not familiar with the dividend issuance and distribution because they are lacking of physical management experience.

This research also analyzed student's referencing frequency of these 6 financial statements (as shown in Table III). The result indicated that the referencing frequency of college students for the following statements, i.e. industrial background, business situation statement, balance sheet, and income statement, is more than that of the master-degree students. The reason for this is that the information required for the R\&D development budget, marketing budget and planned production quantity in these 8 business decisionmaking items should be sourced from the aforesaid four financial statements. In the meantime, the master-degree students presented more market climate information and it is because that the master-degree students considered that the decision should be made after learning the overall market environment.

\section{B. Analysis of Problem Solving Process}

Based on these four steps of comprehension problem, plan, select and execute relating to the problem solving process as proposed by Simon (1960), the number of student's decision-making is calculated for the aforesaid 8 business decision-making items (as shown in Table IV). The purpose is to study the relationship between both groups so as to understand the difference of college students and master-degree students in problem solving and thinking.

It indicated that most of the master-degree students will not only control over key messages but also know about the overall direction and then prioritize key decisions in order to complete the execution. In the meantime, all of college and master-degree respondents are involved in "Plan $\rightarrow$ execution" and "Execution $\rightarrow$ plan" steps. From "Select $\rightarrow$ execution" step, we learned that college students tend to create more options because they are indecisive in how to set these 8 decision-making items at the first timing.

TABLE I: REALM OF MAJOR STATISTICS

\begin{tabular}{llll}
\hline Measure & College students & Master-degree students & Percentage \\
\hline Marketing management & 10 & 7 & $63 \%$ \\
Human resource management & 1 & 0 & $3.7 \%$ \\
Research \& development management & 2 & 0 & $7.4 \%$ \\
Financial management & 1 & 3 & $14.8 \%$ \\
Other & 1 & 2 & $11.1 \%$ \\
Total & 15 & 12 & $100 \%$ \\
\hline
\end{tabular}


TABLE II: ANALYSIS OF BUSINESS DECISION-MAKING SEQUENTIAL

\begin{tabular}{|c|c|c|c|c|c|c|c|c|}
\hline \multirow{2}{*}{ Business decision-making items } & \multicolumn{8}{|c|}{ Decision-making sequential } \\
\hline & $1 \mathrm{st}$ & 2nd & $3 \mathrm{rd}$ & 4th & 5 th & 6th & 7th & 8 th \\
\hline Product pricing & 6 & 3 & 3 & 1 & 1 & 5 & 8 & 0 \\
\hline Planned production quantity & 4 & 4 & 6 & 1 & 5 & 7 & 0 & 0 \\
\hline Equipment investment budget & 2 & 3 & 3 & 6 & 4 & 1 & 8 & 0 \\
\hline $\mathrm{R} \& \mathrm{D}$ budget & 9 & 2 & 7 & 5 & 1 & 0 & 2 & 1 \\
\hline Quantity of material purchased & 0 & 2 & 3 & 5 & 9 & 5 & 2 & 1 \\
\hline Maintenance expense budget & 0 & 2 & 2 & 5 & 4 & 7 & 5 & 2 \\
\hline
\end{tabular}

TABLE III: REFERENCING FREQUENCY OF FINANCIAL STATEMENTS

\begin{tabular}{|c|c|c|c|c|}
\hline \multirow{2}{*}{ Financial statements items } & \multicolumn{2}{|c|}{ College students } & \multicolumn{2}{|c|}{ Master-degree students } \\
\hline & Total & Percentage & Total & Percentage \\
\hline Industrial background & 31 & $56.4 \%$ & 24 & $43.6 \%$ \\
\hline Market climate information & 9 & $36 . \%$ & 16 & $64.0 \%$ \\
\hline Business situation statement & 21 & $55.3 \%$ & 17 & $44.7 \%$ \\
\hline Statement of cash flows & 12 & $50.0 \%$ & 12 & $50.0 \%$ \\
\hline Balance sheet & 14 & $58.3 \%$ & 10 & $41.7 \%$ \\
\hline Income statement & 30 & $63.8 \%$ & 17 & $36.2 \%$ \\
\hline
\end{tabular}

TABLE IV: VERBAL CODING SUMMARY

\begin{tabular}{lllll}
\multicolumn{4}{c}{ TABLE IV: VERBAL CODING SUMMARY } \\
& $\begin{array}{l}\text { Comprehension } \\
\text { problem }\end{array}$ & Plan & Select & Execution \\
\hline Comprehension problem & - & $0 ; 0$ & $0 ; 0$ & $0 ; 0$ \\
Plan & $7 ; 3$ & - & $2 ; 0$ & $15 ; 12$ \\
Select & $4 ; 3$ & $2 ; 0$ & - & $4 ; 0$ \\
Execution & $4 ; 5$ & $15 ; 12$ & $7 ; 3$ & - \\
\hline
\end{tabular}

*The values on the left-side represent college students. The values on the right-side represent master-degree students.

As such, they would list up the items that can be easily comprehended and then select the desired one. Further, some college students are also involved in "Execution $\rightarrow$ select" step, but not for the master-degree students. Based on verbal data file, we discovered that when establishing 8 decision-making items, college students would repeatedly check which items should be created first. However, such situation is not seen in master-degree students and it is because that they are able to control the progress of current decisions and the decision-making procedure.

\section{Analysis of Group Difference}

In this research, the graphical representation method is used to explain the inter-relations between college students and master-degree students in problem solving. The results indicated that college students require more complicated thinking steps. When a problem emerges, they would make the planning for one of the decision-making items and would execute the planned item only and then repeat such process over and over again. When planning certain decision-making items, they would also select and execute the remaining decision-making items based on their own experience. In view of this, college students are lacking a fixed kind of thinking process because they tend to complete these 8 decision-making items according to the situation and the thinking mode that they have experienced at that time (as shown in Figure 1). In comparison, master-degree students are able to execute the decision after comprehending the experiment issues. This should be attributed to their learning experience during normal days and more profound and wider knowledge being accumulated over time. For this reason, they are able to absorb the available information more quickly. Further, they have also built up a set of problem-solving method that allows them to execute the decision and set up such decision-making item directly (as shown in Figure 2). After comparing these two groups, we learned that master-degree students employ simpler thinking logic in problem solving, but college students would need several steps during their thinking process in order to make the decision. As such, college students are less familiar with the problem encountered when serving as a decision maker and on the other, they would rely on intuition to conduct the thinking and judgment when setting up decisions.

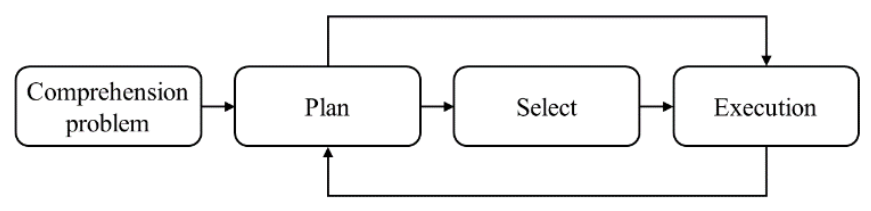

Figure 1 Inter-relations for college student's problem solving process

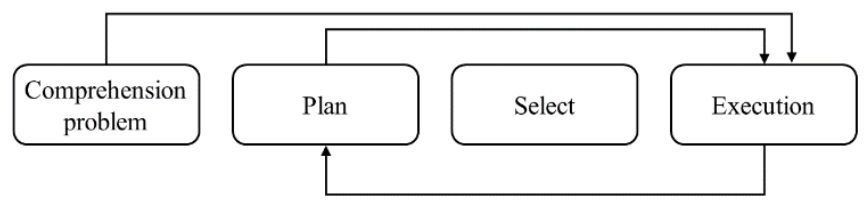

Figure 2 Inter-relations for master-degree student's problem solving process

\section{CONCLUSIONS}

To understand a student's decision-making process, the BOSS is used in this research as the experimental design tool. In the meantime, the protocol analysis of cognitive psychology is also adopted to study the difference between college students and master-degree students in thinking process of decision-making and in problem solving.

The research indicated that when making the decision, most students are not taking their specialty background or realm of major in priority setting. As such, the specialty background or realm of their major will not affect the decision-making sequence. When setting the difference among the 8 decision-making items, college students are more easily influenced by the referencing information instead of focusing on the entire company. In comparison, more diversified factors will be considered by master-degree 
students where they tend to make consideration according to the overall operation approaches and future development as to fulfill the sustainable operation conception.

During comprehension problem stage for problemsolving, college students would review the subject repeatedly that they are unable to control over key messages more efficiently. As for master-degree students, they used to preclude the routine kind of messages and then think about how to set up and plan the required steps. During the plan stage, college students are less familiar with the status of a problem where a loosening kind of structure is established between correlated problems. As for master-degree students, they do not only comprehend deeper about the information content but are also able to control over the information correlativity and implement the strategies. During the select stage, college students are lacking a comprehensive kind of assessment for the execution method and they would carry out the sorting and thinking superficially. In comparison, master-degree students are able to control over the configured solutions and they have established a set of automatic problem solving methods. During execution stage, college students tend to use simplified method and attempt to expand the known messages and then execute directly. Instead, master-degree students are more systematic that they tend to accumulate the knowledge according to the correlativity between individual matters and knowledge.

\section{REFERENCES}

Anderson, J. R. (1990). Cognitive psychology and its implications (3rd Eds.). New York, NY: Freeman.

Chi, M. T. H., \& Glaser, R. (1985). Problem-solving ability. In R. J. Sternberg (Ed.), Human abilities: An information-processing approach (pp. 227-250). New York, NY: Freeman.

Deek, F. P., \& McHugh, J. A. (2000). Problem-solving methodologies and the development of critical thinking skills. Journal of Computer Science Education, 14(1), 6-12.

Eckersley, M. (1988). The form of design processes: A protocol analysis study. Design Studies, 9(2), 86-94.

Gagne, R. M. (1985). The conditions of learning (4th Eds.). New York, NY: Holt, Rinehart \& Winston.

Leckie, G. J., Pettigrew, K. E., \& Sylvai, C. (1996). Modeling the information seeking of professionals: A general model derived from research on engineers, health care professionals, and lawyers, Library Quarterly, 66(2), 161-193.

Lin, N., \& Garvey, W. D. (1972). Information needs and uses. Annual Review of Information Science and Technology, 7, 5-37.

MacDuffie, J. P. (1997). The road to 'root cause': Shop-floor problemsolving at three auto assembly plants. Management Science, 43(4), 479-502.

Mayer, R. E. (1992). Thinking, problem solving, cognition. New York, NY: W.H. Freeman and Company Press.

Simon, H. A. (1960). The new science of management. New York, NY: Harper and Row.

Simon, H. A. (1992). The science of the artificial. Cambridge, MA: MIT Press.

Simon, H. A. (1997). Administrative behavior (4th Eds.). New York, NY: Free Press.

Vessey, I. (1991). Cognitive fit: A theory-based analysis of the graphs versus tables literature. Decision Science, 22(2), 219-240. 